\title{
Article \\ Magnetized black hole as an accelerator of charged particle
}

\author{
Bobur Turimov ${ }^{1,2,3,4}$ (D)
}

1 Ulugh Beg Astronomical Institute, Astronomicheskaya 33, Tashkent 100052, Uzbekistan; bturimov@astrin.uz

4 Akfa University, Kichik Halqa Yuli Street 17, Tashkent 100095, Uzbekistan

3 Research Centre for Theoretical Physics and Astrophysics, Institute of Physics, Silesian University in Opava, Bezručovo nám. 13, CZ-74601 Opava, Czech Republic

4 Webster University in Tashkent, Alisher Navoiy 13, Tashkent 100011, Uzbekistan

\begin{abstract}
Astrophysical accretion processes near the black hole candidates, such as active galactic nuclei (AGN), X-ray binary (XRB), and other astrophysical sources, are associated with highenergetic emission of radiation of relativistic particles and outflows (winds and/or jets). It is widely believed that the magnetic field plays a very important role to explain such high energetic processes in the vicinity of those astrophysical sources. In the present research note, we propose that the black hole is embedded in an asymptotically uniform magnetic field. We investigate the dynamical motion of charged particles in the vicinity of a weakly magnetized black hole. We show that in the presence of the magnetic field, the radius of the innermost stable circular orbits (ISCO) for a charged particle is located close to the black hole's horizon. The fundamental frequencies, such as Keplerian and epicyclic frequencies of the charged particle are split into two parts due to the magnetic field, as an analog of the Zeeman effect. The orbital velocity of the charged particle measured by a local observer has been computed in the presence of the external magnetic field. We also present an analytical expression for the four-acceleration of the charged particle orbiting around black holes. Finally, we determine the intensity of the radiating charged accelerating relativistic particle orbiting around the magnetized black hole.
\end{abstract}

Keywords: magnetized black hole; fundamental frequancies; synchrootron radiation.

\section{Introduction}

Observation evidence of astrophysical black holes, such supermassive black hole $(\mathrm{SMBH})$ and stellar black hole provides new motivation to investigate charged particle dynamics around black hole in the presence of the external electromagnetic field. It is generally accepted that a magnetic field is considered one of main sources of the most energetic processes around supermassive black holes at the center of galaxies, playing the role of "feeder" of the supermassive black hole by trapping dust near the galaxy's center [1].

Synchrotron radiation is, a relativistic case of cyclotron radiation, characterized by emitting photons due to the acceleration of charged particles in the external magnetic field. In a flat space, radiation from a rapidly moving charge and synchrotron radiation (magnetic bremsstahlung) from charged particle moving along circular trajectory arbitrary relativistic velocity in uniform magnetic field has been investigated in [2]. These facts provide new motivations for investigating of radiation from charged particles in the framework of general relativity (GR). It is worth notice that according to "no-hair theorem", the black hole can not possess magnetic field. However, the external magnetic field around the black hole can be generated by its accretion disc, or a sourunded rotating matter, or a companion in binary systems containing neutron star (NS) or/and magnetar with strong magnetic field. One of simple model of magnetized black hole has performed by Wald [3], and similar physical scenarios on magnetized black hole have been considered later, e.g., in [4-14]. According to this model, the black hole immersed 
in an asymptotically uniform external magnetic field that is small enough to change its spacetime grometry. In the Ref. [15], it is shown that the external magnetic field $B$ is negligibally small than the critical magnetic field $B_{M}$ that can infulience the spacetime of the black hole and satisfies the following condition:

$$
B \ll B_{M} \sim 10^{19}\left(\frac{M_{\odot}}{M}\right) \mathrm{G} .
$$

According to the Ref. [16] the magnetic field strength around supermassive black hole $(\mathrm{SMBH})$ is order of $\sim 10^{2} \mathrm{G}$, while in the vicinity of the stellar black hole (SBH), it is about $\sim 10^{4} \mathrm{G}$. The energy of the emitted photon through the cyclotron frequency around SMBH then estimated as

$$
E_{B}=\hbar \omega_{B}=\frac{\hbar q B}{m c} \simeq 1.2 \times 10^{-4}\left(\frac{q}{e}\right)\left(\frac{m}{m_{e}}\right)^{-1}\left(\frac{B}{10^{4} \mathrm{G}}\right) \mathrm{eV},
$$

while around $\mathrm{SBH}$, it has

$$
E_{B} \simeq 1.2\left(\frac{q}{e}\right)\left(\frac{m}{m_{e}}\right)^{-1}\left(\frac{B}{10^{8} \mathrm{G}}\right) \mathrm{eV} .
$$

A complete detailed analysis of interaction between black hole and magnetic field generated by the accretion disc or companion object (it can be neutron star or magnetar with strong magnetic field) is complicated problem which requires numerical magnetohydrodynamic (MHD) simulations [17]. However, approximative methods are also very useful to draw picture of this phenomenon, by considering stationary magnetized black hole solutions in general relativity as was done by Wald [3] and Ernst [18].

The discussing of an interaction between charged particle and electromagnetic fields is very interesting topic from theoretical and observational point of view. A comprehensive physical aspects of the theory of black holes in an external electromagnetic field are reviewed in [19-22]. In the papers [23,24] propagation of scalar field in the background of strongly magnetized black hole (or Ernst spacetime) has been studied and later it is considered for the massive scalar field in [25]. It is shown that in the presence of the strong magnetic field the quasinormal modes are longer lived and have larger oscillation frequencies in both massless and massive scalar fields [26,27]. The effect of the magnetic field in optical properties of black hole has been discussed in Refs. [28,29].

The paper is organized as follows. In Sec. 2, we provide basic necessary equations related to charged test particle motion around the Schwarzschild black hole in the presence of the electromagnetic field. In Sec. 3, we investigate a general description to derive the fundamental frequencies for charged particle orbiting around static black hole, described by arbitrary spacetime with given stationary, spherical-symmetric metric coefficients and electromagnetic fields. Later on, we derived the fundamental frequencies, such as, Keplerian, Larmor and epicyclic frequencies of charge particle around magnetized Schwarzschild black hole. In next Sec. 4, we discuss synchrotron radiation from relativistic charged particle orbting around the magnetized black hole. Finally, in Sec. 5, we summarize found results and give a future outlook related to this work. 


\section{Charged particle dynamics}

In this section, we provide equations of motion for charged particle around the black hole immersed in the uniform magnetic field. In Boyer-Lindquist coordinates $x^{\alpha}=(t, r, \theta, \phi)$, the Schwarzschild metric is given by ${ }^{1}$

$$
d s^{2}=-\left(1-\frac{2 M}{r}\right) d t^{2}+\left(1-\frac{2 M}{r}\right)^{-1} d r^{2}+r^{2}\left(d \theta^{2}+\sin ^{2} \theta d \phi^{2}\right),
$$

51 where $M$ is total mass of the black hole.

The configuration of the electromagnetic field near the black hole has explicitly shown in [3], the non-zero component of the vector potential is given by

$$
A_{\alpha}=\frac{1}{2} \delta_{\alpha}^{\phi} B r^{2} \sin ^{2} \theta
$$

52 where $B$ is the uniform magnetic field strength.

The dynamical motion of charged particle of the mass $m$ and charge $q$ is governed by the following non-geodesic equation

$$
\frac{d U^{\alpha}}{d \lambda}+\Gamma_{\mu \nu}^{\alpha} U^{\mu} U^{v}=\frac{q}{m} F_{\beta}^{\alpha} U^{\beta}, \quad U_{\alpha} U^{\alpha}=-1,
$$

where $U^{\alpha}=d x^{\alpha} / d \lambda$ is the four-velocity of the test particle, $\lambda$ is an affine parameter, $\Gamma_{\mu \nu}^{\alpha}$ are the Christoffel symbols and $F_{\alpha \beta}=A_{\beta, \alpha}-A_{\alpha, \beta}$ is the electromagnetic field tensor. The conserved quantities, namely, the specific energy $\mathcal{E}$, and specific angular momentum $\mathcal{L}$ of charged particle measured at the infinity, can be easily found as

$$
g_{t t} U^{t}=-\mathcal{E}, \quad g_{\phi \phi} U^{\phi}+\frac{q}{m} A_{\phi}=\mathcal{L} .
$$

Using the normalization of four-velocity of the test particle, one can have the following expression:

$$
g_{t t}\left(U^{t}\right)^{2}+g_{r r}\left(U^{r}\right)^{2}+g_{\theta \theta}\left(U^{\theta}\right)^{2}+g_{\phi \phi}\left(U^{\phi}\right)^{2}=-1,
$$

and hereafter introducing the spatial components of the velocity of particle measured by a local observer

$$
v_{r}=\sqrt{-\frac{g_{r r}}{g_{t t}}} \frac{d r}{d t}, \quad v_{\theta}=\sqrt{-\frac{g_{\theta \theta}}{g_{t t}}} \frac{d \theta}{d t}, \quad v_{\phi}=\sqrt{-\frac{g_{\phi \phi}}{g_{t t}}} \frac{d \phi}{d t} .
$$

with the total velocity $v^{2}=v_{r}^{2}+v_{\theta}^{2}+v_{\phi}^{2}$, the expression for the specific energy of charged particle can be expressed as

$$
\mathcal{E}=\sqrt{-\frac{g_{t t}}{1-v^{2}}}=\sqrt{\frac{1-\frac{2 M}{r}}{1-v^{2}}} .
$$

Note that the energy expression (10) is obviously independent of the external magnetic field, however, the radius $r$ and velocity $v$ of charged particle depend on the external magnetic field. One can easily see from the expression (10) that absence the black hole's mass, i.e. $M=0$, the classical expression for the energy of relativistic particle can be obtained as follows, $E=m c^{2}\left(1-v^{2} / c^{2}\right)^{-1 / 2}$.

\footnotetext{
1 Throughout the paper we use the geometric system of units $c=G=\hbar=1$ and spacelike signature $(-,+,+,+)$. However, we restore constants when we compare the obtained results with observational data
} 


\section{Fundamental frequency of charged particle}

Hereafter using normalization of the four-velocity of the test particle i.e. $U^{\alpha} U_{\alpha}=$ -1 , taking into account the expressions (7), one can obtain

$$
g_{r r}\left(U^{r}\right)^{2}+g_{\theta \theta}\left(U^{\theta}\right)^{2}+V(r, \theta)=0,
$$

where

$$
V(r, \theta)=1+\frac{\mathcal{E}^{2}}{g_{t t}}+\frac{1}{g_{\phi \phi}}\left(\mathcal{L}-\frac{q}{m} A_{\phi}\right)^{2} .
$$

As one can see from the expression for the potential (12) for a charged particle one needs the explicit form of the vector potential, while for magnetized particle depends on the components of the magnetic field which means that if we wish to consider both the charged and magnetized particle in the presence of external magnetic field then we need the expressions for the vector potential and components of the magnetic field.

It is interesting to consider the periodic motion of the charged particle orbiting around the black hole which allows determining the fundamental frequencies such as Keplerian and Larmor frequencies. The simple way of deriving the expressions for thus frequencies is to consider motion in the stable circular orbit with, $U^{\alpha}=\left(U^{t}, 0,0, U^{\phi}\right)$, which allows writing

$$
\begin{aligned}
& U^{t}=\frac{1}{\sqrt{-g_{t t}-\Omega^{2} g_{\phi \phi}}}, \\
& \mathcal{E}=-\frac{g_{t t}}{\sqrt{-g_{t t}-\Omega^{2} g_{\phi \phi}}}, \\
& \mathcal{L}=\frac{\Omega g_{t t}}{\sqrt{-g_{\phi \phi}-\Omega^{2} g_{\phi \phi}}}+\frac{q}{m} A_{\phi} .
\end{aligned}
$$

where $\Omega=d \phi / d t$ is the angular velocity of the orbital motion measured by a distant observer.

It is important to determine radius of the innermost stable circular orbit (ISCO) for charged particle. The ISCO radius can be easily determined from the following conditions:

$$
V(r, \theta)=0, \quad \partial_{r} V(r, \theta)=\partial_{\theta} V(r, \theta)=0, \quad \partial_{r}^{2} V(r, \theta) \geq 0, \quad \partial_{\theta}^{2} V(r, \theta) \geq 0 .
$$

Considering charged particle motion in the vicinity of the Schwarzschild black in the presence of the uniform magnetic field, we found that the ICSO radii for both positively and negatively charged particles decrease due to the external uniform magnetic field. Similarly, careful numerical analyses show that the radius of the marginally bound orbit, where the energy of the particle in circular orbit will be the same as its rest energy, or $\mathcal{E}=1$, for charged particle also decrease due to the effect of the external magnetic field. Figure 1 shows the dependence of the ISCO and MBO radii from the magnetic coupling parameter $q B M / m$.

Another important quantity in particle dynamics is the energy efficiency (or, sometimes, it iscalled gravitational deffect mass), calculation of the energy efficiency reather simple i.e. $\eta=1-\mathcal{E}_{\text {ISCO }}$. Our numerical calculations show that the energy efficiency for positively charged particle raeches upto $99.938 \%$ (but, never reaches upto $100 \%$ ), while for negatively charged particle it reaches up to $26.844 \%$. Figure 2 illustrates dependence of the energy efficiency from the magnetic coupling parameter $q B M / m$.

It is also interesting to produce the trajectories of the charged particle orbiting around the magnetized black hole. As we mentioned before that charged particle moves 


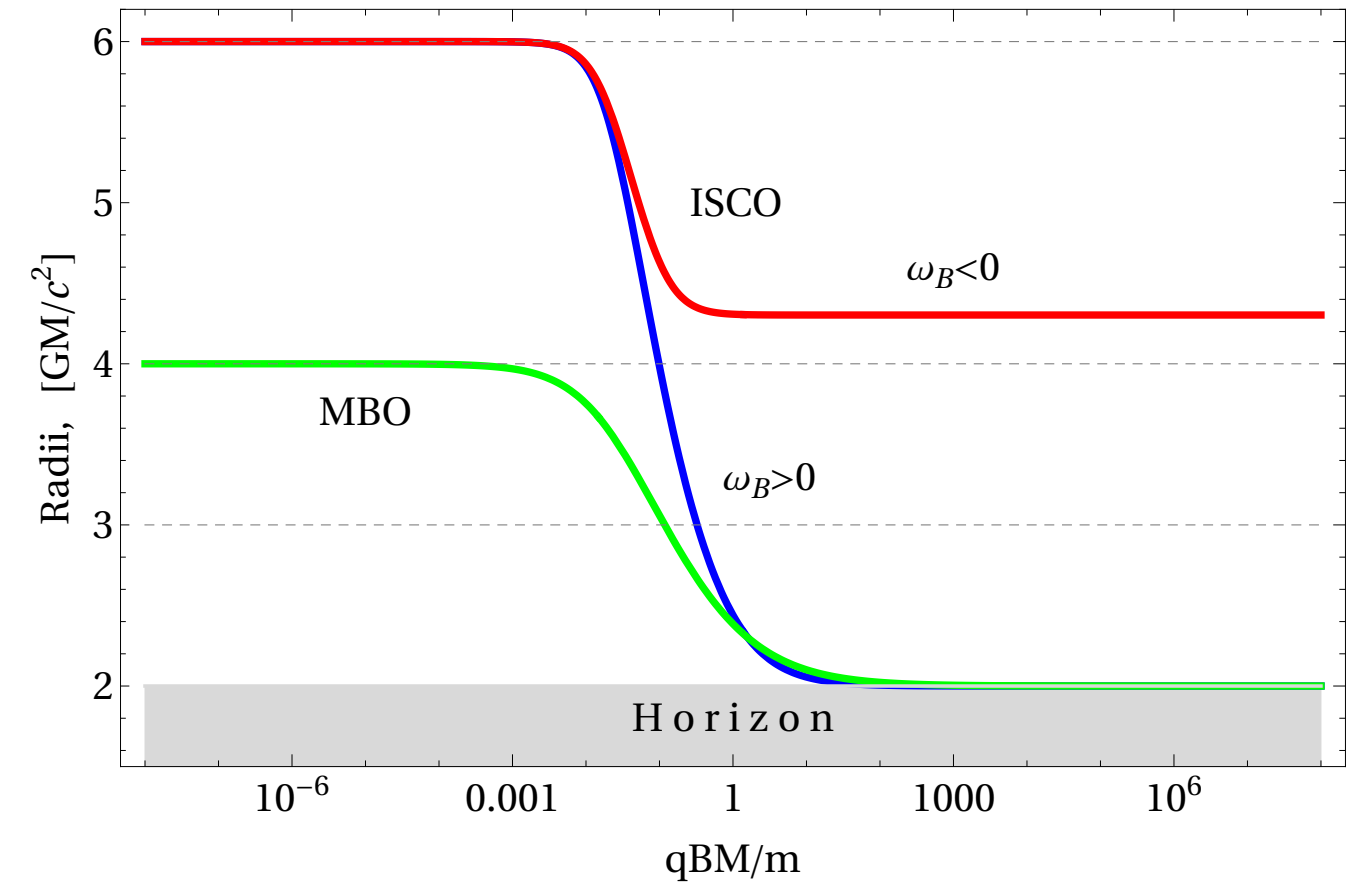

Figure 1. Dependence the ISCO and MBO radii from the magnetic coupling parameter $q B M / m$ for the positivle and negatively charged particle.

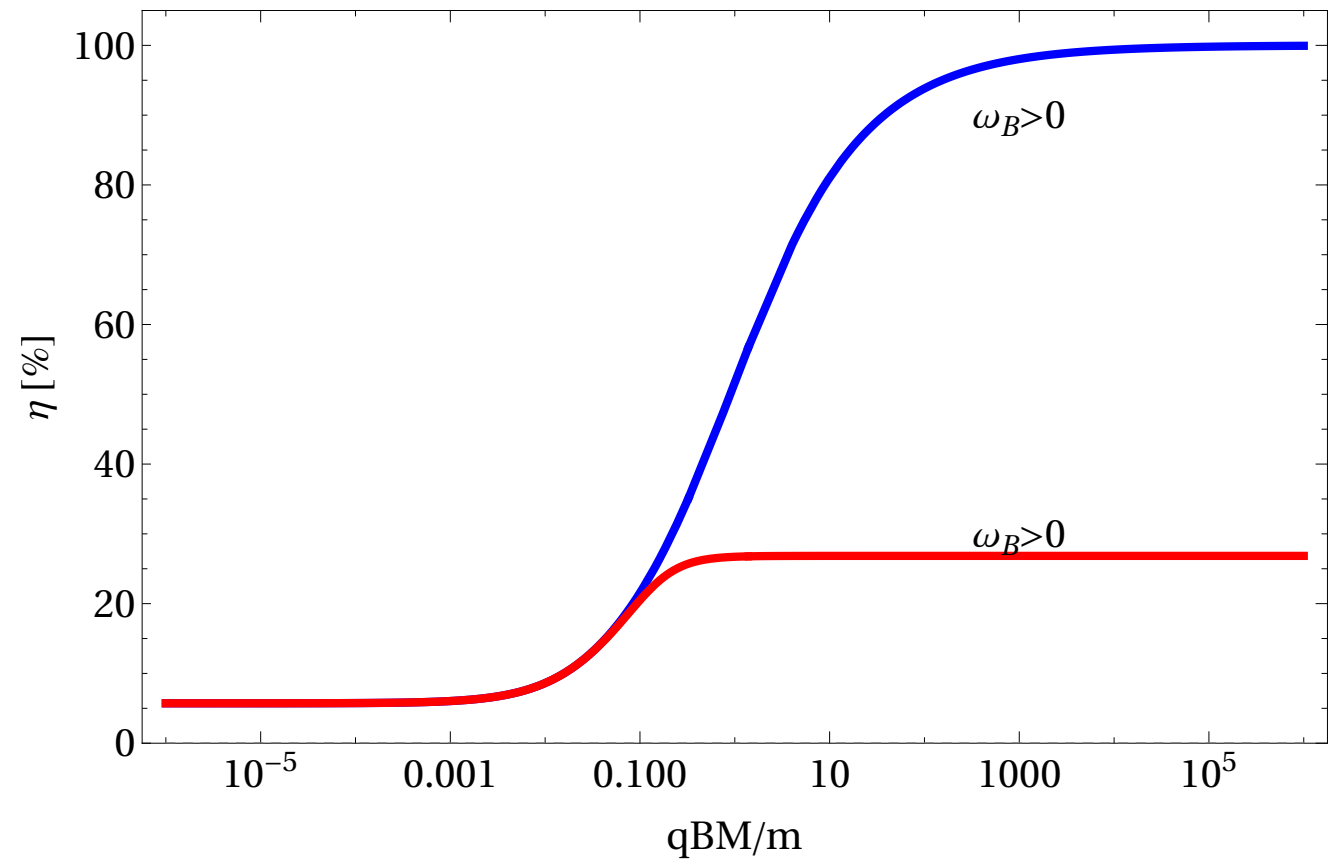

Figure 2. Dependence the energy efficiency from the magnetic coupling parameter $q B M / m$ for the positively and negatively charged particle. 

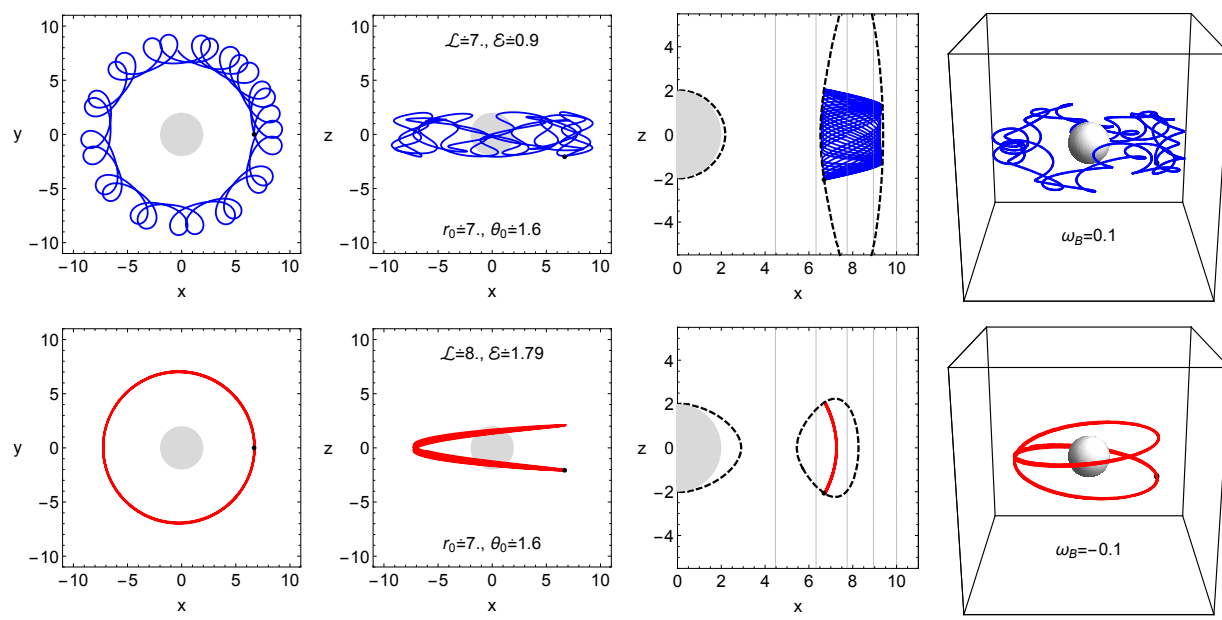

Figure 3. The trajectory of charged particle around magnetized black hole.

in the gravitational and magnetic field and motion is governed by the four second order equations as shown in (6). In order to construct particle trajectory one needs eight initial conditions, two of them can be eliminated using the conserved quantities, due to symmetry another two condition can be written as $t(0)=\phi(0)=0$, while normalization of the four-velocity eliminates one of conditions. Then, we only need to give initial position of particle with random velocity. Figure 3 illustrates the trajectories of charged particle orbiting around magnetized black hole.

\subsection{Keplerean and Larmor frequency}

Now we focus on the derivation of the expression for the orbital angular frequencies, such as Keplerian, and Larmor frequencies, of the charged particle orbiting around the black hole. To do this, let us again consider motion in circular orbit with, $U^{\alpha}=$ $U^{t}(1,0,0, \Omega)$. In the case, from (6), equations for radial and vertical motion can be written

$$
g_{t t, r}+\Omega^{2} g_{\phi \phi, r}=-\frac{2 q}{m} \frac{F_{r t}}{U^{t}}, \quad \Omega g_{\phi \phi, \theta}=-\frac{2 q}{m} \frac{F_{\theta \phi}}{U^{t}} .
$$

Note that the physical meaning of the quantity $\Omega$ above equations are different and this difference can be easily shown by considering neutral particle motion i.e., $q=0$. In this case the solution of the first equation in (17) becomes $\Omega_{0}=\sqrt{-g_{t t, r} / g_{\phi \phi, r}}$ which represents Keplerian frequency for neutral particle. On the other hand solution of the second equation of (17) vanishes $\Omega=0$ for neutral particle, so that non-trivial solution can be found only for charged particle in the presence of external electromagnetic field. In order to find the explicit expressions for Keplerian and Larmor frequencies, one has to eliminate $U^{t}$ by inserting the expression (13) into (17), and after performing simple algebraic manipulations one can obtain

$$
\begin{aligned}
& \Omega^{2}=\Omega_{0}^{2}-\frac{g_{\phi \phi}}{2}\left(\frac{2 q F_{r t}}{m g_{\phi \phi, r}}\right)^{2} \pm \frac{2 q F_{r t}}{m g_{\phi \phi, r}} \sqrt{-g_{t t}-\Omega_{0}^{2} g_{\phi \phi}+\frac{g_{\phi \phi}^{2}}{4}\left(\frac{2 q F_{r t}}{m g_{\phi \phi, r}}\right)^{2}}, \\
& \Omega_{L}= \pm \frac{\sqrt{-g_{t t}}}{\sqrt{g_{\phi \phi}+\left(\frac{m g_{\phi \phi, \theta}}{2 q F_{\theta \phi}}\right)^{2}}} .
\end{aligned}
$$

\subsection{The epicyclic frequencies}

It is also interesting to determine the epicyclic frequencies $\left(\Omega_{r}, \Omega_{\theta}\right)$ produced by oscillatory motion of charged particle along radial and vertical direction at stable circular 
orbit around black hole in the presence of external magnetic field. Here we study quasiperiodic oscillation of charged particle around given stable circular orbit. Before move on further that we expand of the function $V(r, \theta)$ in the form

$$
\begin{aligned}
V(r, \theta) & =V\left(r_{0}, \theta_{0}\right)+\left.\delta r \partial_{r} V(r, \theta)\right|_{x_{0}}+\left.\delta \theta \partial_{\theta} V(r, \theta)\right|_{x_{0}} \\
& +\left.\frac{1}{2} \delta r^{2} \partial_{r}^{2} V(r, \theta)\right|_{x_{0}}+\left.\frac{1}{2} \delta \theta^{2} \partial_{\theta}^{2} V(r, \theta)\right|_{x_{0}}+\left.\delta r \delta \theta \partial_{r} \partial_{\theta} V(r, \theta)\right|_{x_{0}}+\mathcal{O}\left(\delta r^{3}, \delta \theta^{3}\right) \\
& \left.\simeq \frac{1}{2} \delta r^{2} \partial_{r}^{2} V(r, \theta)\right|_{x_{0}}+\left.\frac{1}{2} \delta \theta^{2} \partial_{\theta}^{2} V(r, \theta)\right|_{x_{0}}{ }^{\prime}
\end{aligned}
$$

where $x_{0}=\left(r_{0}, \theta_{0}\right)$ are the stationary points. Here we have used the conditions (??)-(??). Now inserting the expression (12) into (11), using the expression (20) one can obtain equation of harmonic oscillatory motion for charged particle, around the stationary orbit $\left(r_{0}, \theta_{0}\right)$, for the displacement $\delta_{r}=r-r_{0}, \delta_{\theta}=\theta-\theta_{0}$ in the form:

$$
\frac{d^{2}}{d t^{2}} \delta_{r}+\Omega_{\mathrm{r}}^{2} \delta_{r}=0, \quad \frac{d^{2}}{d t^{2}} \delta_{\theta}+\Omega^{2} \delta_{\theta}=0,
$$

where the epicyclic frequencies in (21) can be calculated by

$$
\left(\Omega_{r}^{2}, \Omega_{\theta}^{2}\right)=\frac{1}{2\left(U^{t}\right)^{2}}\left(\frac{1}{g_{r r}} \partial_{r}^{2} V(r, \theta), \frac{1}{g_{\theta \theta}} \partial_{\theta}^{2} V(r, \theta)\right) .
$$

Finally, using the equations (12)-(15) the explicit form of the epicyclic frequencies $\left(\Omega_{r}, \Omega_{\theta}\right)$ of charged particle orbiting around black hole can be expressed as

$$
\begin{aligned}
g_{i i} \Omega_{i}^{2} & =\frac{\left(g_{t t, i}\right)^{2}}{g_{t t}}-\frac{1}{2} g_{t t, i i}+\Omega^{2}\left(\frac{\left(g_{\phi \phi, i}\right)^{2}}{g_{\phi \phi}}-\frac{1}{2} g_{\phi \phi, i i}\right) \\
& -\frac{q}{m} \Omega\left(A_{\phi, i i}-2 A_{\phi, i} \frac{g_{\phi \phi, i}}{g_{\phi \phi}}\right) \sqrt{-g_{t t}-\Omega^{2} g_{\phi \phi}}-\left(\frac{q}{m}\right)^{2} A_{\phi, i}^{2}\left(\Omega^{2}+\frac{g_{t t}}{g_{\phi \phi}}\right),
\end{aligned}
$$

where $i=(r, \theta)$. From equation (23) one can see that the radial and vertical frequencies $\left(\Omega_{r}, \Omega_{\theta}\right)$ depend on the the background geometry, the external magnetic field and also parameters of the test particle. Once background spacetime geometry and external magnetic field are given then one can immediately determine $\Omega_{r}$ and $\Omega_{\theta}$, however keep in mind that they still depend Keplerian frequency which is most important in the calculation of the fundamental frequencies.

Using the general expressions (18), (19), and (23) for the fundamental frequencies such as Keplerian, epicyclic and Larmor frequencies of charged particle orbiting around Schwarzschild blach immersed in uniform magnetic field can be expressed as

$$
\begin{aligned}
& \Omega^{2}=\Omega_{0}^{2}\left[1+\frac{f}{2} \frac{\omega_{B}^{2}}{\Omega_{0}^{2}} \pm \frac{\omega_{B}}{\Omega_{0}} \sqrt{\frac{f^{2}}{4} \frac{\omega_{B}^{2}}{\Omega_{0}^{2}}+f-\Omega_{0}^{2} r^{2}}\right]^{-1}, \\
& \Omega_{r}^{2}=3 \Omega^{2} f\left[1+\frac{\omega_{B}}{\Omega} \sqrt{f-\Omega^{2} r^{2}}+\frac{\omega_{B}^{2}}{3 \Omega^{2}}\left(f-\Omega^{2} r^{2}\right)\right]-2 \Omega_{0}^{2}, \\
& \Omega_{\theta}^{2}=\Omega^{2}\left(1+\frac{\omega_{B}}{\Omega} \sqrt{f-\Omega^{2} r^{2}}\right), \\
& \Omega_{L}^{2}=f \frac{\omega_{B}^{2}}{1+\omega_{B}^{2} r^{2}},
\end{aligned}
$$

where $\omega_{B}=q B / m$ is the cyclotron frequency for charged particle and $\Omega_{0}=\sqrt{M / r^{3}}$ is Keplerian frequency for neutral particle in Schwarzschild space. Absence of the external 

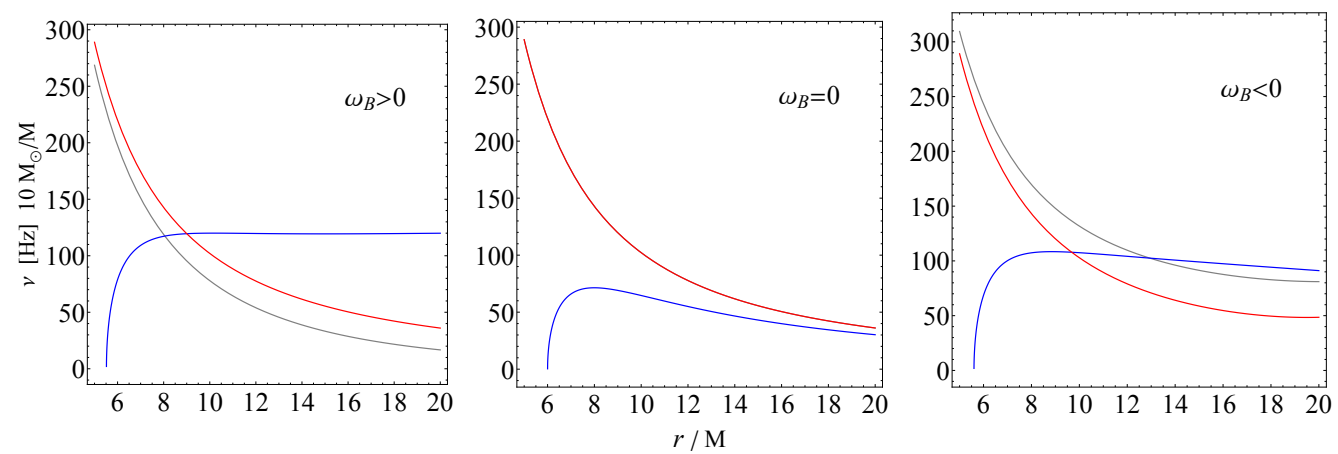

Figure 4. Radial dependence of the fundamental frequencies for for positive $\omega_{B}>0$, zero $\omega_{B}=0$ and negative $\omega_{B}<0$ values of the interaction parameter. The gray line represents Keplerian frequency, while the blue and red lines are responsible frequencies for the radial and vertical oscillations.

uniform magnetic field, i.e. $B=0$ or $\omega_{B}=0$, the expressions for the fundamental frequencies take the form:

$$
\Omega=\Omega_{0}=\sqrt{\frac{M}{r^{3}}}, \quad \Omega_{r}=\Omega_{0} \sqrt{1-\frac{6 M}{r}}, \quad \Omega_{\theta}=\Omega_{0}=\sqrt{\frac{M}{r^{3}}}, \quad \Omega_{L}=0 .
$$

Notice that angular frequencies, $\Omega_{i}$, is related to the frequencies, $v_{i}$, as follows $\Omega_{i}=2 \pi v_{i}$. To have an idea about order of magnitude of these frequencies, one can estimate the orbital frequency of test particle for a Schwarzschild black hole in the form:

$$
v=220\left(\frac{10 M_{\odot}}{M}\right)\left(\frac{6 M}{r}\right)^{3 / 2} \mathrm{~Hz} .
$$

It is worth noting that these frequencies can be observed distance greater that the ISCO position of test particle around the black hole. Figure 4 draws radial dependence of the fundamental frequencies for positive, negative and zero values of the interaction parameter. As we can see from Fig. 4 that near the ISCO position the values of the fundamental frequencies of charged particle are almost same as for neutral particle. While for a large distance they are dramatically changed due to the external magnetic field, in particular, the radial frequency increases at the distance far from the central object.

\section{Synchrotron radiation by magnetized black hole}

Now we focus on investigating of synchrotron radiation from relativistic charged particle in the vicinity of magnetized Schwarzschild black hole. According to the Ref. [2], the expression for the four-momentum loss of the accelarating test particle can be written as

$$
\frac{d P^{\alpha}}{d \lambda}=\frac{2 q^{2}}{3} w_{\beta} w^{\beta} U^{\alpha}, \quad \rightarrow \quad U^{\alpha} \frac{d P_{\alpha}}{d \lambda}=-\frac{2 q^{2}}{3} w_{\beta} w^{\beta} .
$$

It is well-known that accelerating relativistic charged particle emits radiation. Now we concentrate on the radiation of the accelerating charged particle orbiting around the black hole. The radiation spectrum of the relativistic charged particle in curved spacetime can be expressed as [2]

$$
I=\frac{2 q^{2}}{3} w_{\alpha} w^{\alpha}
$$


where $w^{\alpha}$ is the four-acceleration of particle in a curved space defined as $w^{\alpha}=U^{\beta} \nabla_{\beta} U^{\alpha}$, on the other hand taking account non-geodesic equation (6), one can write

$$
w^{\alpha}=\frac{q}{m} F_{\beta}^{\alpha} U^{\beta}, \quad w_{\alpha} U^{\alpha} \equiv 0 .
$$

For simplicity, we consider the motion of charged particle in the stable circular orbit with $U^{\alpha}=U^{t}(1,0,0, \Omega)$ and to see the behavior of the radiation spectrum. Since the velocity and acceleration of particle are orthogonal to each other, i.e., $w_{\alpha} U^{\alpha} \equiv 0$, we can immediately express the four-acceleration of particle in the form, $w^{\alpha}=\left(0, w^{r}, w^{\theta}, 0\right)$, where the components of the acceleration can be defined as

$$
\begin{aligned}
& w_{r}=\frac{q \Omega F_{r \phi}}{m \sqrt{-g_{t t}-\Omega^{2} g_{\phi \phi}}}, \\
& w_{\theta}=\frac{q \Omega F_{\theta \phi}}{m \sqrt{-g_{t t}-\Omega^{2} g_{\phi \phi}}} .
\end{aligned}
$$

Finally, the expressions for the intensity (31) of the radiating accelerated charged particle orbiting around magnetized black hole is

$$
I=-\frac{2 q^{4}}{3 m^{2}} \frac{1}{g_{t t}+\Omega^{2} g_{\phi \phi}}\left(g^{r r} F_{r \phi}^{2}+g^{\theta \theta} F_{\theta \phi}^{2}\right),
$$

Similarly, one can also consider more realistic situation that the charged particle falling into black hole with the four-velocity, $U^{\alpha}=U^{t}(1, u, 0, \omega)$, where $u=d r / d t$ is radial velocity and $\omega=d \phi / d t$ is angular velocity of particle. In this case, from the condition $w_{\alpha} U^{\alpha}=0$, one can argue that the radial acceleration of charged particle vanishes $w^{r}=0$, however, vertical acceleration still should be exist, i.e., $w^{\theta} \neq 0$. Finally, the intensity of charged particle can be expressed as

$$
I=-\frac{2 q^{4}}{3 m^{2}}\left(\frac{\omega^{2} g^{\theta \theta} F_{\theta \phi}^{2}}{g_{t t}+u^{2} g_{r r}+\omega^{2} g_{\phi \phi}}\right),
$$

which concludes that accreting charged particle onto magnetized black hole emits the electromagnetic radiation.

\section{Summary}

The study of black holes analyzing the observed data on the accretion disc may be helpful to investigate the electromagnetic radiation in the vicinity of compact objects. In the present research work, we have investigated the motion of charged particle and the energetic process, namely, the fundamental frequencies and synchrotron radiation by the magnetized Schwarzschild black hole. The main results of the paper can be summarized as follows:

In this paper, we have done analyses for the effects of the charge coupling parameter on the ISCO parameters representing the specific energy, specific angular momentum, the critical angle, and the innermost radius, respectively. To that end, we have written the equations of motion for a charged particle in the vicinity of the magnetized Schwarzschild black hole and then have numerically calculated the ISCO parameters according to the standard way of derivation for the charged particles. Numerical results show that the ISCO radius for charged particle may change non-monotonously with its charge parameter $\omega_{B}$. As a result, we can see that particles owning identical charges but different mass may degenerate into one ISCO depending on the external magnetic field.

Investigations for particle motion in the vicinity of the black hole can provide valuable references for the study of astrophysical events, for example, quasiperiodic oscillations and high energy events relating to the fundamental frequencies of a test 
particle orbiting around the astrophysical black hole. Our result about the degeneration of the particle orbits may provide a useful theoretical prediction of the observation of the electromagnetic waves. Thinking of the magnetized black hole spacetime, one can envisage that two particles endowed with identical mass but opposite electrical charge move at the same ISCO in the same directions and have an elastic collision with each other, resulting in other two particles with the same spin but less charge, then both of these two particles will have a smaller ISCO orbit after the instantaneous event. We suspect that there must be an astrophysically observable phenomenon corresponding to this interesting collisional event.

Finally, we investigate synchrotron radiation from the acceleration of charged particle in the vicinity of the magnetized Schwarzschild black hole. The explicit expressions for the intensity of radiating charged relativistic particles around the magnetized black hole due to acceleration by electromagnetic (Coulomb and Lorentz) forces have been derived. Numerical calculations show that the radiation intensity of accelerated charged particle around the magnetized black hole is in order of $\sim 10^{35} \mathrm{Erg} / \mathrm{s}$. Another important result shows that radially falling charged particle onto the black hole possess vertical acceleration which means in that case it can emit electromagnetic radiation.

It is also interesting to study charged particle acceleration around rotating magnetized black hole (magnetized Kerr black hole). The effects from the rotation of the black hole and the external magnetic field in synchrotron radiation by an accelerated charged particle. The following investigations are underway.

Funding: This research was funded by Grants No. VA-FA-F-2-008 and No. MRB-AN-2019-29 of the Uzbekistan Ministry for Innovative Development and by the internal grant SGS/12/2019 of Silesian University in Opava, Czech Republic.

Data Availability Statement: No data available in this article.

Acknowledgments: The author thanks M. Kološ for valuable discussions and comments.

Conflicts of Interest: The author declares no conflict of interest.

\section{Abbreviations}

Throughout the paper the following abbreviations are used:

$\begin{array}{ll}\text { AGN } & \text { Active galactic nuclei } \\ \text { XRB } & \text { X-ray binary } \\ \text { SMBH } & \text { Supermasssive black hole } \\ \text { SBH } & \text { Stellar black hole } \\ \text { ISCO } & \text { Innermost stable circular orbit }\end{array}$

\section{References}

1. Lopez-Rodriguez, E.; Antonucci, R.; Chary, R.R.; Kishimoto, M. The Highly Polarized Dusty Emission Core of Cygnus A. Astrophys. J. Lett. 2018, 861, L23, [1806.11114]. doi:10.3847/2041-8213/aacff5.

2. Landau, L.D.; Lifshitz, E.M. The Classical Theory of Fields, Course of Theoretical Physics, Volume 2; Elsevier Butterworth-Heinemann: Oxford, 2004.

3. Wald, R.M. Black hole in a uniform magnetic field. Phys. Rev. D. 1974, 10, 1680-1685. doi:10.1103/PhysRevD.10.1680.

4. Kološ, M.; Stuchlík, Z.; Tursunov, A. Quasi-harmonic oscillatory motion of charged particles around a Schwarzschild black hole immersed in a uniform magnetic field. Classical and Quantum Gravity 2015, 32, 165009, [arXiv:gr-qc/1506.06799]. doi:10.1088/02649381/32/16/165009.

5. Toshmatov, B.; Abdujabbarov, A.; Ahmedov, B.; Stuchlík, Z. Motion and high energy collision of magnetized particles around a Hořava-Lifshitz black hole. Astrophys. Space Sci. 2015, 360, 19. doi:10.1007/s10509-015-2533-y.

6. Stuchlík, Z.; Kološ, M. Acceleration of the charged particles due to chaotic scattering in the combined black hole gravitational field and asymptotically uniform magnetic field. Eur. Phys. J. C 2016, 76, 32, [arXiv:gr-qc/1511.02936]. doi:10.1140/epjc/s10052015-3862-2.

7. Tursunov, A.; Stuchlík, Z.; Kološ, M. Circular orbits and related quasiharmonic oscillatory motion of charged particles around weakly magnetized rotating black holes. Phys. Rev. D 2016, 93, 084012, [arXiv:gr-qc/1603.07264]. doi:10.1103/PhysRevD.93.084012. 
8. Kološ, M.; Tursunov, A.; Stuchlík, Z. Possible signature of the magnetic fields related to quasi-periodic oscillations observed in microquasars. Eur. Phys. J. C 2017, 77, 860, [arXiv:astro-ph.HE/1707.02224]. doi:10.1140/epjc/s10052-017-5431-3.

9. Al Zahrani, A.M.; Frolov, V.P.; Shoom, A.A. Critical escape velocity for a charged particle moving around a weakly magnetized Schwarzschild black hole. Phys. Rev. D 2013, 87, 084043, [arXiv:gr-qc/1301.4633]. doi:10.1103/PhysRevD.87.084043.

10. Abdujabbarov, A.; Ahmedov, B.; Rahimov, O.; Salikhbaev, U. Magnetized particle motion and acceleration around a Schwarzschild black hole in a magnetic field. Phys. Scripta 2014, 89, 084008. doi:10.1088/0031-8949/89/8/084008.

11. Abdujabbarov, A.A.; Ahmedov, B.J.; Jurayeva, N.B. Charged-particle motion around a rotating non-Kerr black hole immersed in a uniform magnetic field. Phys. Rev. D 2013,87, 064042. doi:10.1103/PhysRevD.87.064042.

12. Turimov, B. Electromagnetic fields in vicinity of tidal charged static black hole. International Journal of Modern Physics D 2018, 27, 1850092. doi:10.1142/S021827181850092X.

13. Shaymatov, S.; Ahmedov, B.; Stuchlík, Z.; Abdujabbarov, A. Effect of an external magnetic field on particle acceleration by a rotating black hole surrounded with quintessential energy. Int. J. Mod. Phys. D 2018, 27, 1850088. doi:10.1142/S0218271818500888.

14. Benavides-Gallego, C.A.; Abdujabbarov, A.; Malafarina, D.; Ahmedov, B.; Bambi, C. Charged particle motion and electromagnetic field in $\gamma$ spacetime. Phys. Rev. D 2019, 99, 044012. doi:10.1103/PhysRevD.99.044012.

15. Frolov, V.P.; Shoom, A.A. Motion of charged particles near a weakly magnetized Schwarzschild black hole. Phys. Rev. D 2010, 82, 084034, [arXiv:gr-qc/1008.2985]. doi:10.1103/PhysRevD.82.084034.

16. Piotrovich, M.Y.; Silant'ev, N.A.; Gnedin, Y.N.; Natsvlishvili, T.M. Magnetic Fields of Black Holes and the Variability Plane. ArXiv e-prints 2010, [arXiv:astro-ph.CO/1002.4948].

17. McKinney, J.C.; Tchekhovskoy, A.; Blandford, R.D. General relativistic magnetohydrodynamic simulations of magnetically choked accretion flows around black holes. MNRAS 2012, 423, 3083-3117, [arXiv:astro-ph.HE/1201.4163]. doi:10.1111/j.13652966.2012.21074.x.

18. Ernst, F.J. Black holes in a magnetic universe. J. Math. Phys. 1976, 17, 54-56. doi:10.1063/1.522781.

19. Aliev, A.N.; Gal'Tsov, D.V. “Magnetized” black holes. Sov. Phys. Usp. 1989, 32, 75.

20. Kokkotas, K.D.; Konoplya, R.A.; Zhidenko, A. Quasinormal modes, scattering, and Hawking radiation of Kerr-Newman black holes in a magnetic field. Phys. Rev. D 2011, 83, 024031, [arXiv:gr-qc/1011.1843]. doi:10.1103/PhysRevD.83.024031.

21. Turimov, B.; Rayimbaev, J.; Abdujabbarov, A.; Ahmedov, B.; Stuchlík, Z. Test particle motion around a black hole in EinsteinMaxwell-scalar theory. Phys. Rev. D 2020, 102, 064052, [arXiv:gr-qc/2008.08613]. doi:10.1103/PhysRevD.102.064052.

22. Turimov, B.; Rahimov, O.; Ahmedov, B.; Stuchlík, Z.; Boymurodova, K. Dynamical motion of matter around a charged black hole. International Journal of Modern Physics D 2021, 30, 2150037-407. doi:10.1142/S0218271821500371.

23. Konoplya, R.A.; Fontana, R.D.B. Quasinormal modes of black holes immersed in a strong magnetic field. Phys. Lett. B 2008, 659, 375-379, [arXiv:hep-th/0707.1156]. doi:10.1016/j.physletb.2007.10.065.

24. Konoplya, R.A. Superradiant instability for black holes immersed in a magnetic field. Phys. Lett. B 2008, 666, 283-287, [arXiv:hep-th/0801.0846]. doi:10.1016/j.physletb.2008.07.079.

25. Wu, C.; Xu, R. Decay of massive scalar field in a black hole background immersed in magnetic field. Eur. Phys. J. C 2015, 75, 391, [arXiv:gr-qc/1507.04911]. doi:10.1140/epjc/s10052-015-3632-1.

26. Brito, R.; Cardoso, V.; Pani, P. Superradiant instability of black holes immersed in a magnetic field. Phys. Rev. D 2014, 89, 104045, [arXiv:gr-qc/1405.2098]. doi:10.1103/PhysRevD.89.104045.

27. Turimov, B.; Toshmatov, B.; Ahmedov, B.; Stuchlík, Z. Quasinormal modes of magnetized black hole. Phys. Rev. D 2019, 100, 084038, [arXiv:gr-qc/1910.00939]. doi:10.1103/PhysRevD.100.084038.

28. Turimov, B.; Ahmedov, B.; Abdujabbarov, A.; Bambi, C. Gravitational lensing by a magnetized compact object in the presence of plasma. International Journal of Modern Physics D 2019, 28, 2040013-187, [arXiv:gr-qc/1802.03293]. doi:10.1142/S0218271820400131.

29. Ahmedov, B.; Turimov, B.; Stuchlík, Z.; Tursunov, A. Optical properties of magnetized black hole in plasma. International Journal of Modern Physics Conference Series, 2019, Vol. 49, International Journal of Modern Physics Conference Series, p. 1960018. doi:10.1142/S2010194519600188. 